\section{PERFUSION MAPPING IN HYPERTROPHIC CARDIOMYOPATHY: MICROVASCULAR DYSFUNCTION OCCURS REGARDLESS OF HYPERTROPHY}

${ }^{1}$ Claudia Camaioni, ${ }^{1,2}$ Kristopher Knott, ${ }^{1,3,4}$ Gabriella Captur, ${ }^{1}$ Anish Bhuva, 1,2Luis Lopes, ${ }^{5}$ Hui Xue, ${ }^{6}$ Louise Brown, ${ }^{1}$ Saidi Mohiddin, ${ }^{1,2}$ Charlotte Manisty, ${ }^{6}$ Sven Plein, ${ }^{5}$ Peter Kellman, 1,2,4 James C Moon. ${ }^{1}$ Barts Heart Centre, The Cardiovascular Magnetic Resonance Imaging Unit and The Inherited Cardiovascular Diseases Unit, St Bartholomew's Hospital, West Smithfield, London, UK; ${ }^{2}$ Institute of Cardiovascular Science, University College London, London, UK; ${ }^{3}$ NIHR University College London Hospitals Biomedical Research Centre, London, UK; ${ }^{4} U C L$ Biological Mass Spectrometry Laboratory, Institute of Child Health and Great Ormond Street Hospital, London, UK; ${ }^{5}$ National Heart, Lung, and Blood Institute, National Institutes of Health, DHHS, Bethesda, MD, USA; ${ }^{6}$ Division of Biomedical Imaging, LICAMM, University of Leeds, Clarendon Way, Leeds, UK

\subsection{6/heartinl-2017-311399.4}

Aims Patients with hypertrophic cardiomyopathy (HCM) histologically have myocyte hypertrophy and disarray, small vessel disease and fibrosis. The links and sequencing of these abnormalities in phenotype development is unclear. We implemented in-line perfusion mapping in HCM undergoing stress cardiovascular resonance (CMR) and measured pixel by pixel flow at stress and rest, in order to exactly quantify the microvascular dysfunction.

Methods $30 \mathrm{HCM}$ patients and 10 controls, age and gendermatched, referred for stress CMR due to atypical chest pain and having normal findings, underwent adenosine stress perfusion CMR using in-line perfusion mapping in Gadgetron to deliver pixel-by-pixel myocardial blood flow (MBF, $\mathrm{mls} / \mathrm{g} / \mathrm{min}$ ) for three short axis views ( \pm an additional long axis) at stress and rest. Transmural (tmMBF), endocardial (endoMBF) and epicardial (epiMBF) were calculated for each LV segment Figure). MBF was correlated with segmental wall thickness (WT) and late gadolinium enhancement (LGE), quantified by the 5 SD method.

Results Stress MBF was lower in HCM (2.23 \pm 0.74 vs 3.49 $\pm 0.82 \mathrm{~mL} / \mathrm{g} / \mathrm{min}, p 0.008$ for tmMBF). This was most marked for endoMBF. The reductions were associated with increasing wall thickness (endoMBF R: $-0.43, \mathrm{p}<0.001$ ) and the amount of segmental LGE (endoMBP R: $-0.27, \mathrm{p}<0.001$ ). MBF could actually fall with stress compared to rest - a myocardial perfusion reserve less than one - this was more common with increasing hypertrophy. Rest MBF was the same in HCM and controls. Apparently normal HCM segments (no LVH, no LGE) had significantly lower stress MBF compared to controls (tmMBF $\mathrm{p}=0.001$, endoMBF $\mathrm{p}=0.0002$, epiMBF $\mathrm{p}=0.071$ ).

Conclusion Perfusion mapping confirms that HCM patients have reduced MPR that is linked to wall thickness and scar. Stress flow can be lower than rest in hypertrophied segments. Apparently normal segments (no LVH, no LGE) have abnormal MBF suggesting this may be an early marker of the HCM phenotype. Further studies are needed (e.g. in carriers).

\section{MULTIMODALITY ASSESSMENT OF RISK IN DILATED CARDIOMYOPATHY- THE IMPORTANCE OF CMR}

${ }^{1,2} \mathrm{U}$ Tayal, ${ }^{3} \mathrm{~S}$ Newsome, ${ }^{2} \mathrm{I}$ Voges, ${ }^{1} \mathrm{~N}$ Whiffin, ${ }^{2} \mathrm{R}$ Buchan, ${ }^{2} \mathrm{~B}$ Halliday, ${ }^{2} \mathrm{~A}$ Lota, ${ }^{2} \mathrm{C}$ Izgi, ${ }^{1,2} \mathrm{PJ}$ Barton, ${ }^{2} \mathrm{R}$ Baruah, ${ }^{2} \mathrm{~J}$ Jarman, ${ }^{2} \mathrm{M}$ Frenneaux, ${ }^{1,2} \mathrm{DJ}$ Pennell, ${ }^{1,2} \mathrm{JS}$ Ware, ${ }^{1,4} \mathrm{SA}$ Cook,

$1,2 \mathrm{SK}$ Prasad. ${ }^{1}$ National Heart Lung Institute, Imperial College London, UK; ${ }^{2}$ Royal Brompton Hospital, London, UK; ${ }^{3}$ Department of Medical Statistics, London School of Hygiene and Tropical Medicine, UK; ${ }^{4}$ Duke National University Hospital, Singapore

\subsection{6/heartjnl-2017-311399.5}

Background Dilated cardiomyopathy (DCM) has a 20\% 5 year mortality. Cardiac MR (CMR) is an established outcome predictor. We evaluate the additive role of novel genetic and circulating biomarkers.

Purpose Perform an integrated assessment to evaluate the prognostic importance of CMR parameters in DCM in the context of clinical, genetic and biomarker data.

Methods Prospectively recruited DCM patients underwent comprehensive clinical evaluation, CMR with late-gadolinium enhancement (LGE), sequencing for rare variants in major DCM genes (titin-TTNtv, myosin heavy chain-MYH7, troponin T2-TNNT2, lamin-LMNA), biomarker assessment of BNP,

Abstract 004 Figure 1 Perfusion map in apical HCM. Long axis (far left) and basal-mid-apical short axis slices (to the right) at stress (upper panel) and rest (lower panel). There is a circumferential endocardial perfusion defect in the apical segments at stress. Each pixel represents myocardial blood flow (MBF). MBF falls in the apical segments during stress. 
troponin I (hsTnI), Galectin 3 and ST2, and follow up for clinical events. Cox proportional hazard modelling evaluated the primary composite endpoint of cardiovascular death, major arrhythmic events (aborted sudden cardiac death, appropriate ICD activation, sustained ventricular tachycardia, ventricular fibrillation) and major heart failure events (heart transplant, left ventricular assist device, unplanned heart failure hospitalisation).

Results In total, 423 patients with DCM (mean age 53.6 \pm 14.1 years, $67 \%$ male) were followed up for a median of 4.0 years (IQR 2.1-5.8). Mean left ventricular ejection fraction (LVEF) was $40 \% \pm 12.5 \%$. One third of patients had midwall fibrosis (MWF-LGE) $(\mathrm{n}=137,32 \%)$. Mean indexed left atrial volume (LAVi) was $61 \pm 27.6 \mathrm{mls}$.

On genetic analysis, 53 patients $(12.5 \%)$ had TTNtv, 14 patients $(3.3 \%)$ had non-truncating variants in MYH7, 5 patients $(1.2 \%)$ had non-truncating variants in TNNT2 and 5 patients $(1.2 \%)$ had LMNA variants.

In total, 44 patients $(10.4 \%)$ met the primary endpoint. On multivariable analysis, an optimal model predicting the primary endpoint was built without genetic or biomarker data (Table 1). This consisted of LVEF, LAVi, and MWF-LGE (Table 2).

On univariable analysis, BNP, Galectin 3 and hsTnI were associated with the primary endpoint (Table 2). When the novel variables were added to the optimised model, Galectin 3 and LMNA variants were predictive of the primary endpoint (Table 3). In the final adjusted model, CMR parameters remained predictive of the primary endpoint. Of these, MWFLGE was the strongest predictor (adjusted HR 2.12 (1.064.02), $\mathrm{p}=0.02$ ).

Conclusion On integrated multi-modality risk stratification in DCM, while circulating and genetic biomarkers improve risk stratification, CMR parameters remain strong independent predictors of outcome.

Acknowledgements UT is supported by a Medical Research Council (UK) Clinical Research Training Fellowship. The study has also been supported by the Rosetrees Foundation, the Alexander Jansons Foundation, the Wellcome Trust and the NIHR Cardiovascular Biomedical Research Unit of Royal Brompton and Harefield NHS Foundation Trust and Imperial College London.

Abstract 005 Table 1 Variables evaluated in building baseline Cox proportional hazard model predicting primary endpoint

\begin{tabular}{|c|c|c|}
\hline & Variables evaluated & Retained \\
\hline Clinical & $\begin{array}{l}\text { Age, Gender, Ethnicity, NYHA class*, a family history of DCM*, a } \\
\text { family history of sudden cardiac death, a history of sustained* or } \\
\text { non sustained* ventricular tachycardia, a history of atrial } \\
\text { fibrillation, diabetes, hypertension*, left bundle branch block, } \\
\text { resting heart rate, beta blocker use, aldosterone antagonist use, } \\
\text { diuretic use*, ACE inhibitor use }\end{array}$ & \\
\hline CMR & $\begin{array}{l}\text { LVEF}^{*}, \text { RVEF}^{*} \text {, LAVi* } \\
\text { LV end systolic volume*, indexed left ventricular mass*, indexed } \\
\text { RV end diastolic volume, indexed RV end systolic volume*, MWF- } \\
\text { LGE* }^{*}\end{array}$ & $\begin{array}{l}\text { LVEF, LAVi, } \\
\text { LGE }\end{array}$ \\
\hline
\end{tabular}

* = Significant on univariable analysis
Abstract 005 Table 2 Results of baseline Cox proportional hazard modelling predicting primary endpoint

\begin{tabular}{lllll}
\hline & Unadjusted analysis & & Adjusted analysis & \\
\hline Variable & Hazard ratio & P value & Hazard ratio & P value \\
& $\begin{array}{l}(95 \% \text { confidence } \\
\text { intervals })\end{array}$ & & $\begin{array}{l}(95 \% \text { confidence } \\
\text { intervals })\end{array}$ & \\
& $0.62(0.50-0.78)$ & $<0.0001$ & $0.65(0.51-0.83)$ & 0.001 \\
LVEF (per 10\%) & & 0.03 & $2.12(1.14-3.92$ & 0.017 \\
$\begin{array}{l}\text { MWF-LGE } \\
\text { present }\end{array}$ & $1.99(1.10-3.60)$ & & & \\
$\begin{array}{l}\text { LAVi (per } \\
10 \mathrm{mls})\end{array}$ & $1.12(1.07-1.17)$ & $<0.0001$ & $1.12(1.06-1.18)$ & $<0.0001$ \\
& & & &
\end{tabular}

LVEF- left ventricular ejection fraction, MWF-LGE - mid-wall fibrosis detected on late gadolinium enhancement imaging, LAVi - indexed left atrial volume

Abstract 005 Table 3 Results of evaluation of biomarkers and genetic variants using Cox proportional hazard modelling predicting primary endpoint

\begin{tabular}{|c|c|c|c|c|}
\hline & Unadjusted analysis & & Adjusted analysis* & \\
\hline Variable & $\begin{array}{l}\text { Hazard ratio } \\
\text { ( } 95 \% \text { confidence } \\
\text { intervals) }\end{array}$ & $P$ value & $\begin{array}{l}\text { Hazard ratio } \\
\text { ( } 95 \% \text { confidence } \\
\text { intervals) }\end{array}$ & $\begin{array}{l}\mathrm{P} \\
\text { value }\end{array}$ \\
\hline BNP (NPX) & $1.42(1.22-1.65)$ & $<0.0001$ & $1.20(0.99-1.47)$ & 0.06 \\
\hline $\begin{array}{l}\text { Galectin-3 (per } \\
1 \mathrm{ng} / \mathrm{ml} \text { ) }\end{array}$ & $1.03(1.01-1.06)$ & 0.016 & $1.04(1.01-1.07)$ & $0.015^{\wedge}$ \\
\hline $\begin{array}{l}\text { Troponin I (per pg/ } \\
\text { ml) }\end{array}$ & $1.01(1.01-1.02)$ & 0.0003 & $1.01(0.99-1.02)$ & 0.19 \\
\hline ST2 (NPX) & $0.97(0.70-1.33)$ & 0.84 & $0.76(0.54-1.07)$ & 0.12 \\
\hline TTNtv present & $0.90(0.38-2.15)$ & 0.81 & $0.88(0.36-2.11)$ & 0.77 \\
\hline MYH7ms present & $0.67(0.09-4.84)$ & 0.69 & 1.37 (0.18 to 10.27$)$ & 0.76 \\
\hline LMNAvar present & $2.84(0.68-11.78)$ & 0.15 & $5.73(1.29-25.46)$ & $0.02^{\wedge}$ \\
\hline
\end{tabular}

LVEF- left ventricular ejection fraction, MWF-LGE - mid-wall fibrosis detected on late gadolinium enhancement imaging, LAVi - indexed left atrial volume, BNP- brain natriuretic peptide, NPX - normalised protein expression value, TTNtv- truncating variant in titin gene, MYH7ms- non-truncating variant in myosin heavy chain gene, LMNAvar- variant in lamin gene. No patients with TNNT2ms (non truncating variant in troponin T2 gene) variants met the primary composite endpoint.

${ }^{*}$ Adjusted for LVEF, LGE, LAVi

$\wedge$ Significant $p<0.05$

Abstract 005 Table 4 Results of final adjusted Cox proportional hazard model predicting primary endpoint

\begin{tabular}{llll}
\hline & Hazard ratio & 95\% confidence interval & P value \\
\hline LVEF (per 10\%) & 0.64 & $(0.5-0.83)$ & 0.001 \\
MWF-LGE present & 2.12 & $(1.12-4.02)$ & 0.021 \\
LAVi (per 10 mls) & 1.12 & $(1.06-1.18)$ & $<0.0001$ \\
LMNAvar present & 6.48 & $(1.44-29.15)$ & 0.015 \\
Galectin-3 (per 1 $\mathrm{ng} / \mathrm{ml})$ & 1.04 & $(1.01-1.07)$ & 0.008
\end{tabular}

LVEF- left ventricular ejection fraction, MWF-LGE - mid-wall fibrosis detected on late gadolinium enhancement imaging, LAVi - indexed left atrial volume, LMNAvar- variant in lamin gene 